\title{
Calibrating the Galaxy Halo-Black Hole Relation Based on the Clustering of Quasars
}

\section{Citation}

Wyithe, J. Stuart B., and Abraham Loeb. 2005. "Calibrating the Galaxy Halo-Black Hole Relation Based on the Clustering of Quasars." The Astrophysical Journal 621 (1): 95-103. https:// doi.org/10.1086/425263.

\section{Permanent link}

http://nrs.harvard.edu/urn-3:HUL.InstRepos:41393420

\section{Terms of Use}

This article was downloaded from Harvard University's DASH repository, and is made available under the terms and conditions applicable to Other Posted Material, as set forth at http:// nrs.harvard.edu/urn-3:HUL.InstRepos:dash.current.terms-of-use\#LAA

\section{Share Your Story}

The Harvard community has made this article openly available.

Please share how this access benefits you. Submit a story.

Accessibility 


\title{
CALIBRATING THE GALAXY HALO-BLACK HOLE RELATION BASED ON THE CLUSTERING OF QUASARS
}

\author{
J. Stuart B. Wyithe ${ }^{1}$ and Abraham Loeb ${ }^{2}$ \\ Received 2004 March 31; accepted 2004 August 9
}

\begin{abstract}
The observed number counts of quasars can be explained either by long-lived activity within rare massive hosts or by short-lived activity within smaller, more common hosts. It has been argued that quasar lifetimes can therefore be inferred from their clustering length, which determines the typical mass of the quasar host. Here we point out that the relationship between the mass of the black hole and the circular velocity of its host dark matter halo is more fundamental to the determination of the clustering length. In particular, the clustering length observed in the TwoDegree Field (2dF) quasar redshift survey is consistent with the galactic halo-black hole relation observed in local galaxies, provided that quasars shine at $\sim 10 \%-100 \%$ of their Eddington luminosity. The slow evolution of the clustering length with redshift inferred in the $2 \mathrm{dF}$ quasar survey favors a black hole mass whose redshiftindependent scaling is with halo circular velocity rather than halo mass. These results are independent from observations of the number counts of bright quasars, which can be used to determine the quasar lifetime and its dependence on redshift. We show that if quasar activity results from galaxy mergers, then the number counts of quasars imply an episodic quasar lifetime that is set by the dynamical time of the host galaxy rather than by the Salpeter time. Our results imply that as the redshift increases, the central black holes comprise a larger fraction of their host galaxy mass, and the quasar lifetime gets shorter.
\end{abstract}

Subject headings: cosmology: observations — cosmology: theory — quasars: general

\section{INTRODUCTION}

The Sloan Digital Sky Survey (SDSS; York et al. 2000) and the Two-Degree Field (2dF) quasar redshift survey (Croom et al. 2001b) have measured redshifts for large samples of quasars and determined their luminosity function over a wide range of redshifts (Boyle et al. 2000; Fan et al. 2001a, 2001b, 2003). The $2 \mathrm{dF}$ survey has also been used to constrain the clustering properties of quasars (Croom et al. 2001a). It has been suggested that quasars have clustering statistics similar to optically selected galaxies in the local universe, with a clustering length $R_{0} \sim 8 \mathrm{Mpc}$. The large sample size of the $2 \mathrm{dF}$ quasars also provided clues about the variation of clustering length with redshift (Croom et al. 2001a) and apparent magnitude (Croom et al. 2002). Quasars appear to be more clustered at high redshift, although with a relatively mild trend. There is also evidence that more luminous quasars may be more highly clustered.

The quasar correlation length determines the typical mass of the dark matter halo in which the quasar resides. As pointed out by Martini \& Weinberg (2001) and Haiman \& Hui (2001), one can therefore derive the quasar duty cycle by comparing the number density of quasars with the density of host dark matter halos. The quasar lifetime then follows from the product of the duty cycle and the lifetime of the dark matter halo in between major mergers, although there is a degeneracy between the lifetime and the quasar occupation fraction or beaming. Preliminary results suggested quasar lifetimes of $t_{q} \sim 10^{6}-10^{7} \mathrm{yr}$, consistent with the values determined by other methods (see Martini 2004 for a review), including the transverse proximity effect (Jakobsen et al. 2003) and counting arguments relative to

\footnotetext{
${ }^{1}$ University of Melbourne, Parkville, VIC 3010, Australia; swyithe@isis. ph.unimelb.edu.au.

${ }^{2}$ Harvard-Smithsonian Center for Astrophysics, 60 Garden Street, Cambridge, MA 02138; aloeb@cfa.harvard.edu.
}

the local population of remnant supermassive black holes (SMBHs; see Yu \& Tremaine 2002). Kauffmann \& Haehnelt (2002) used a detailed semianalytical model (Kauffmann \& Haehnelt 2000) to predict the correlation length of quasars and its evolution with redshift. They found that their model reproduces the correct correlation length as well as its redshift evolution for present-day quasar lifetimes of $t_{q} \sim 10^{7} \mathrm{yr}$.

In this work we argue that the correlation length of quasars is fundamentally determined by the relation between the masses of SMBHs and their host galactic halos rather than by the quasar lifetime. We find that the amplitude of the correlation length depends on the product of the SMBH-halo relation and the typical fraction of the Eddington luminosity at which quasars shine. Moreover, we show that the evolution of the correlation length is sensitive to how the SMBH-halo relation evolves with redshift and therefore to the physics of SMBH formation and quasar evolution. Although groups or clusters of galaxies should contribute to the correlation function of quasars, our discussion focuses on spatial scales much larger than the virial radius of these systems. When comparing our models to data, we consider halo masses well below those of groups, for which the SMBH-halo relation applies.

The paper is organized as follows. In $\S \S 2$ and 3 we discuss the calculation of the correlation function of quasars with a particular apparent magnitude $B$ and the different scenarios for the evolution of the SMBH-galaxy halo relation. In $\S 4$ we compare fiducial model correlation functions to the results of the $2 \mathrm{dF}$ quasar redshift survey (Croom et al. 2001a, 2002). We find that the shallow evolution of the correlation length implies that SMBH mass has a redshift-independent scaling with the circular velocity of the host dark matter halo rather than with its mass. The ranges of the normalizations in the SMBH-galaxy halo relation and of the fraction of the Eddington luminosity allowed by the observed quasar correlation function are explored in $\S 5$. In $\S 6$ we examine the quasar lifetime by requiring 
that observational constraints from both the correlation and luminosity functions be satisfied simultaneously. Finally, in $\S 8$ we summarize our results and discuss a very simple, physically motivated model that satisfies all constraints with no free parameters. Throughout the paper we adopt the set of cosmological parameters determined by the Wilkinson Microwave Anisotropy Probe (WMAP; Spergel et al. 2003), namely mass density parameters of $\Omega_{m}=0.27$ in matter, $\Omega_{b}=0.044$ in baryons, $\Omega_{\Lambda}=0.73$ in a cosmological constant, and a Hubble constant of $H_{0}=71 \mathrm{~km} \mathrm{~s}^{-1} \mathrm{Mpc}^{-1}$.

\section{THE CORRELATION FUNCTION OF QUASARS}

The mass correlation function between halos of masses $M_{1}$ and $M_{2}$ separated by a comoving distance $R$ is (see Scannapieco \& Barkana 2002 and references therein)

$\xi_{m}\left(M_{1}, M_{2}, R\right)=\frac{1}{2 \pi^{2}} \int d k k^{2} P(k) \frac{\sin (k R)}{k R} W\left(k R_{1}\right) W\left(k R_{2}\right)$,

where

$$
R_{1,2}=\left(\frac{3 M_{1,2}}{4 \pi \rho_{m}}\right)^{1 / 3}
$$

$W$ is the window function (top hat in real space), $P(k)$ is the power spectrum, and $\rho_{m}$ is the cosmic mass density. The dark matter halo correlation function for halos of mass $M$ is obtained from the product of the mass correlation function $\xi_{m}(M, M, R)$ and the square of the ratio between the variances of the halo and mass distributions. This ratio, $b$, is defined as the halo bias; its value for a halo mass $M$ can be approximated using the Press-Schechter formalism (Mo \& White 1996), modified to include nonspherical collapse (Sheth et al. 2001),

$b(M, z)=1+\frac{1}{\delta_{c, 0}}\left[\nu^{\prime 2}+b \nu^{\prime 2(1-c)}-\frac{\nu^{\prime 2 c} / \sqrt{a}}{\nu^{\prime 2 c}+b(1-c)(1-c / 2)}\right]$,

where $\delta_{c, 0}(\approx 1.69)$ is the critical overdensity threshold for spherical collapse, $\delta_{c}(z)=\delta_{c, 0} / D(z), D(z)$ is the growth factor at redshift $z, \sigma$ is the variance on a mass scale $M, \nu \equiv$ $\delta_{c}^{2}(z) / \sigma^{2}(M), \nu^{\prime} \equiv \sqrt{a} \nu, a=0.707, b=0.5$, and $c=0.6$. This expression yields an accurate approximation to the halo bias determined from $N$-body simulations (Sheth et al. 2001).

We need the halo correlation function between halos of different masses. The bias (eq. [3]) yields the excess probability relative to the mass distribution of finding a halo at any point. We can therefore determine the halo correlation function for halos of masses $M_{1}$ and $M_{2}$ using the product of the biases for individual masses,

$$
\xi_{h}\left(M_{1}, M_{2}, R\right)=b\left(M_{1}, z\right) b\left(M_{2}, z\right) \xi_{m}\left(M_{1}, M_{2}, R\right) D(z)^{2} .
$$

If the correlation function between halos forming at different redshifts is required, then the more general analytic treatment of Scannapieco \& Barkana (2002) should be used.

We would like to constrain the relationship between $\mathrm{SMBH}$ mass $M_{\mathrm{bh}}$ and galactic halo mass $M_{\text {halo }}$ by constructing a the- oretical quasar correlation function for comparison with observational data. Observations of the quasar correlation function measure the clustering of quasars of a certain luminosity rather than the clustering of halos or SMBHs of a particular mass. We therefore need to associate the $B$-band luminosity $L_{B}$ of a quasar with the halo mass of its host galaxy $M_{\text {halo }}$. We begin by assuming quasars to have a spectral energy distribution corresponding to the median of their population (Elvis et al. 1994). We then allow the SMBH to shine at a fraction $\eta$ of its Eddington luminosity, so that in solar units the $B$-band luminosity of the quasar is

$$
\frac{L_{B}}{L_{B, \odot}}=5.73 \times 10^{12} \eta\left(\frac{M_{\mathrm{bh}}}{10^{9} M_{\odot}}\right) .
$$

We also need to specify a relation between SMBH and halo mass $\left[M_{\text {bh }}=f\left(M_{\text {halo }}\right)\right.$; see $\left.\S 3\right]$. Given a luminosity $L_{B}$, quasar pairs shining with Eddington fractions $\eta_{1}$ and $\eta_{2}$ have their correlation function specified by equation (4), where $M_{1,2}=$ $f^{-1}\left(M_{\mathrm{bh}, 1,2}\right)$, and $M_{\mathrm{bh}, 1,2}=L_{B} /\left(5.73 \times 10^{3} \eta_{1,2} L_{B, \odot}\right) M_{\odot}$.

The correlation function for a population of quasars with a luminosity $L_{B}$ can therefore be computed by drawing pairs of $\eta$-values from an appropriate probability distribution, $d P_{\text {obs }} / d \eta$. The correlation function of quasars with luminosity $L_{B}$ is

$$
\xi_{q}\left(L_{B}\right)=\left\langle\xi_{h}\left(L_{B}, \eta_{1}, \eta_{2}\right)\right\rangle
$$

where $\eta_{1,2}$ are drawn from $d P_{\text {obs }} / d \eta$, and angular brackets denote an average over the probability distribution of $\eta$-values. We note that calculation of the correlation function at a given quasar luminosity requires only specification of the relation between quasar luminosity and halo mass. Other uncertain quantities, like the quasar lifetime, the frequency of the active quasar phase, and the black hole occupation fraction, as well as the dependencies of these quantities on halo mass do not enter the calculation.

We consider two distributions for $\eta$ in this paper. In the first case all quasars shine at their Eddington luminosity, so that $d P_{\text {obs }} / d \eta \propto \delta(\eta-1)$, where $\delta$ is the Dirac delta function. This corresponds to an idealized quasar light curve that is a top-hat function in time, $\eta(t)=\Theta(-t) \Theta\left(t-t_{q}\right)$, whereby the quasar shines at the Eddington luminosity $(\eta=1)$ throughout its lifetime $t_{q}$. In the second case, the observed distribution is assumed be flat in the logarithm of $\eta$, so that $d P_{\text {obs }} / d(\log \eta)$ is constant. This is the form of distribution expected for a quasar light curve of the exponential form $\Theta(-t) \eta(t)=\exp \left(-t / t_{q}\right)$.

\section{THE $M_{\text {bh }}-M_{\text {halo }}$ RELATION}

Dormant SMBHs are ubiquitous in local galaxies (Magorrian et al. 1998). The masses of these SMBHs scale with physical properties of their hosts (e.g., Magorrian et al. 1998; Merritt \& Ferrarese 2001; Tremaine et al. 2002). Motivated by local observations (Ferrarese 2002), we assume a relation $M_{\mathrm{bh}}=$ $f\left(M_{\text {halo }}, z\right)$ between SMBH mass and the mass of the host dark matter halo. Measurement of the $M_{\mathrm{bh}}-M_{\text {halo }}$ relation for local galaxies requires a choice for the relationship between the circular velocity at the radius probed by observations of galaxies and the circular velocity at the virial radius. This relationship depends on the dark matter mass profile and is still uncertain because of the gravitational interaction between the baryons (which cool and often dominate gravity near the center of galaxies) and the dark matter. Ferrarese (2002) presented $M_{\mathrm{bh}}-M_{\text {halo }}$ relations for three different cases (see her Fig. 5). The first case makes the simplifying assumption that the virial velocity of the 
halo represents its circular velocity, as is the case for a singular isothermal sphere. The second case corresponds to the universal profile of Navarro et al. (1996, hereafter NFW96), and the third to the profile derived based on the weak lensing study of Seljak (2002).

The $M_{\mathrm{bh}}-M_{\text {halo }}$ relation may be applied to the calculations of the correlation function of quasars because the masses of SMBHs scale the same way with the physical properties of their host galaxies in both quiescent and active galaxies (McLure \& Dunlop 2002). The function $f$ is constrained by local observations (Ferrarese 2002), and we consider two different forms for its redshift dependence.

Case A.-We assume that SMBH mass is correlated with the halo circular velocity. This scenario is supported empirically by Shields et al. (2003), who studied quasars out to $z \sim 3$ and demonstrated that the relation between $M_{\mathrm{bh}}$ and the stellar velocity dispersion does not evolve with redshift. This is expected if the mass of the black hole is determined by the depth of the gravitational potential well in which it resides, as would be the case if growth is regulated by feedback from quasar outflows (e.g., Silk \& Rees 1998; Wyithe \& Loeb 2003). Expressing the halo circular velocity $v_{c}$ in terms of the halo mass $M$ and redshift $z$, the redshift-dependent relation between the SMBH and halo masses can be written as

$$
\begin{aligned}
M_{\mathrm{bh}}\left(M_{\text {halo }}, z\right) & =\text { const } \times v_{c}^{5} \\
& =\epsilon M_{\text {halo }}\left(\frac{M_{\text {halo }}}{10^{12} M_{\odot}}\right)^{2 / 3}[\zeta(z)]^{5 / 6}(1+z)^{5 / 2},
\end{aligned}
$$

where $\epsilon$ is a constant, $\zeta(z)$ is close to unity and defined as $\zeta \equiv$ $\left[\left(\Omega_{m} / \Omega_{m}^{z}\right)\left(\Delta_{c} / 18 \pi^{2}\right)\right], \Omega_{m}^{z} \equiv\left[1+\left(\Omega_{\Lambda} / \Omega_{m}\right)(1+z)^{-3}\right]^{-1}, \Delta_{c}=$ $18 \pi^{2}+82 d-39 d^{2}$, and $d=\Omega_{m}^{z}-1$ (see eqs. [22]-[25] in Barkana \& Loeb 2001 for more details).

Case B.-We consider the scenario in which the SMBH mass maintains the same dependence on its host halo mass at all redshifts, namely

$$
\begin{aligned}
M_{\mathrm{bh}}\left(M_{\text {halo }}, z\right) & =\text { const } \times M_{\text {halo }}^{5 / 3} \\
& =\epsilon M_{\text {halo }}\left(\frac{M_{\text {halo }}}{10^{12} M_{\odot}}\right)^{2 / 3}[\zeta(0)]^{5 / 6} .
\end{aligned}
$$

The normalizing constant in these relations has an observed value of $\epsilon=\epsilon_{\mathrm{SIS}} \approx 10^{-5.1}$ at $z=0$ (Ferrarese 2002), where the subscript SIS denotes the underlying assumption that the halo mass profile resembles a singular isothermal sphere. For later use we note that the corresponding normalizations assuming an NFW96 profile and the profile found by Seljak (2002) are $\epsilon=$ $\epsilon_{\mathrm{NFW}}=3.7 \epsilon_{\mathrm{SIS}}$ and $\epsilon=\epsilon_{\mathrm{S} 02}=25 \epsilon_{\mathrm{SIS}}$, respectively.

In the following section we construct model correlation functions assuming both of these scenarios and compare the results to observations. We show that case $\mathrm{A}$ is consistent with the data of the $2 \mathrm{dF}$ quasar redshift survey, while case B is not.

\subsection{Summary of Models}

Before proceeding to the comparison with the observed correlation length we label six models of interest:

AI. The fiducial model, $M_{\mathrm{bh}} \propto v_{c}^{5}, \eta=1$, and $\epsilon=\epsilon_{\mathrm{SIS}}$. AII. $M_{\mathrm{bh}} \propto v_{c}^{5}, d P_{\mathrm{obs}} / d(\log \eta)=$ const, and $\epsilon=\epsilon_{\mathrm{SIS}}$. AIII. $M_{\mathrm{bh}} \propto v_{c}^{5}, d P_{\mathrm{obs}} / d(\log \eta)=$ const, and $\epsilon=10 \epsilon_{\mathrm{SIS}}$.
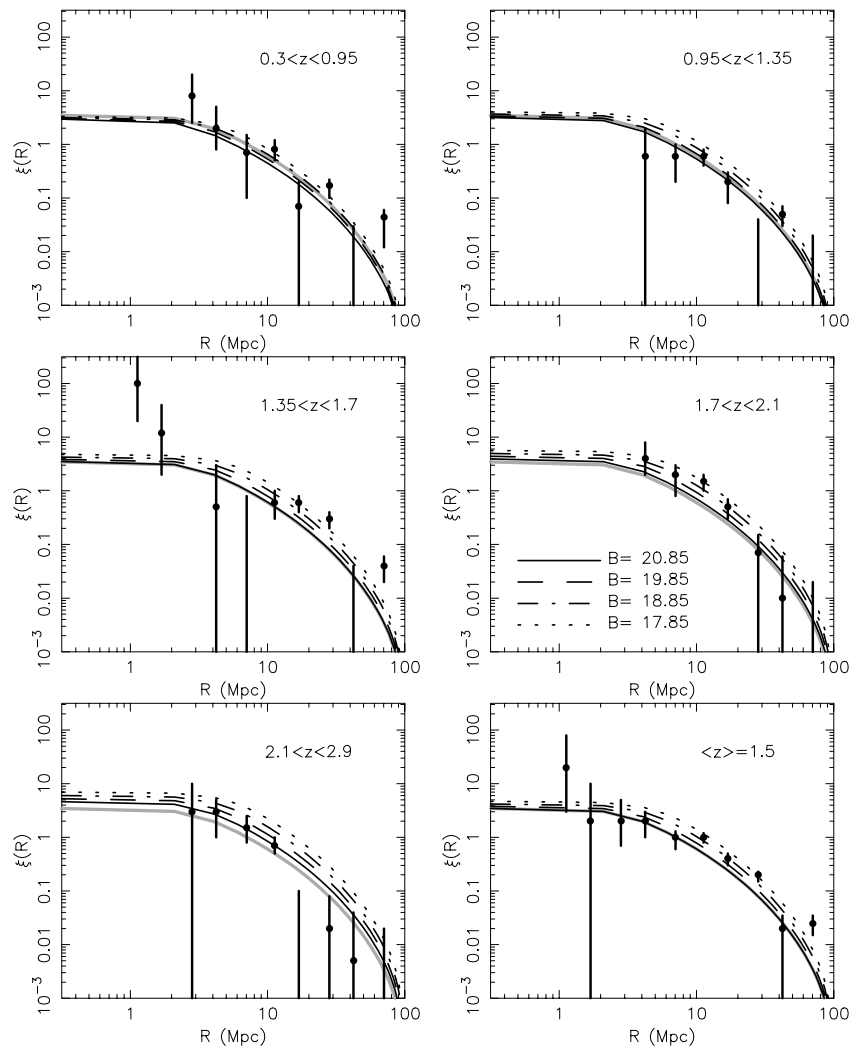

FIG. 1.-Predicted correlation function at various redshifts, in comparison to the $2 \mathrm{dF}$ data (Croom et al. 2001a). The dark lines show the correlation function predictions for quasars of various apparent $B$-band magnitudes. The $2 \mathrm{dF}$ limit is $B \sim 20.85$. The bottom right panel shows data from the entire $2 \mathrm{dF}$ sample in comparison to the theoretical prediction at the mean quasar redshift of $\langle z\rangle=1.5$. The $B=20.85$ prediction at this redshift is also shown by thick gray lines in the other panels to guide the eye. This case assumes $M_{\mathrm{bh}} \propto v_{c}^{5}$, normalized to local observations, and $\eta=1$ (case AI).

BI. $M_{\mathrm{bh}} \propto M^{3 / 2}, \eta=1$, and $\epsilon=\epsilon_{\mathrm{SIS}}$.

BII. $M_{\mathrm{bh}} \propto M^{3 / 2}, d P_{\mathrm{obs}} / d(\log \eta)=$ const, and $\epsilon=\epsilon_{\mathrm{SIS}}$.

BIII. $M_{\mathrm{bh}} \propto M^{3 / 2}, d P_{\mathrm{obs}} / d(\log \eta)=$ const, and $\epsilon=10 \epsilon_{\mathrm{SIS}}$.

In models AII, AIII, BII, and BIII, the range $0.01 \leq \eta \leq 1$ is considered. We refer to the above labels when presenting our results throughout the remainder of the paper.

The models described above use an $M_{\mathrm{bh}}-M_{\text {halo }}$ relation with a normalization derived under the simplifying assumption of a flat rotation curve. However, the circular velocity at the radii probed by observations of galaxies is likely to be larger than the circular velocity at their virial radius. The normalization $\epsilon$ in equations (7) and (8) may therefore be larger than the value $\epsilon_{\mathrm{SIS}}$ considered here (Ferrarese 2002). For any universal halo profile, this difference is degenerate with a simple renormalization of $\eta$. The results in the following section can be applied to a case in which the normalization is $\epsilon^{\prime}=C \epsilon$ by substituting a correspondingly lower value of the Eddington luminosity $\eta^{\prime}=\eta / C$. The allowed range of the product $\epsilon \eta$ is explored in $\S 5$.

\section{COMPARISON WITH OBSERVATIONS}

Next, we compare model correlation functions to observations from the $2 \mathrm{dF}$ quasar redshift survey (Croom et al. 2001a, 2002). Figure 1 shows the correlation function obtained by assuming that SMBH mass scales with halo circular velocity and that all quasars shine at their Eddington luminosity (case AI). We show the predicted correlation function at various redshifts 

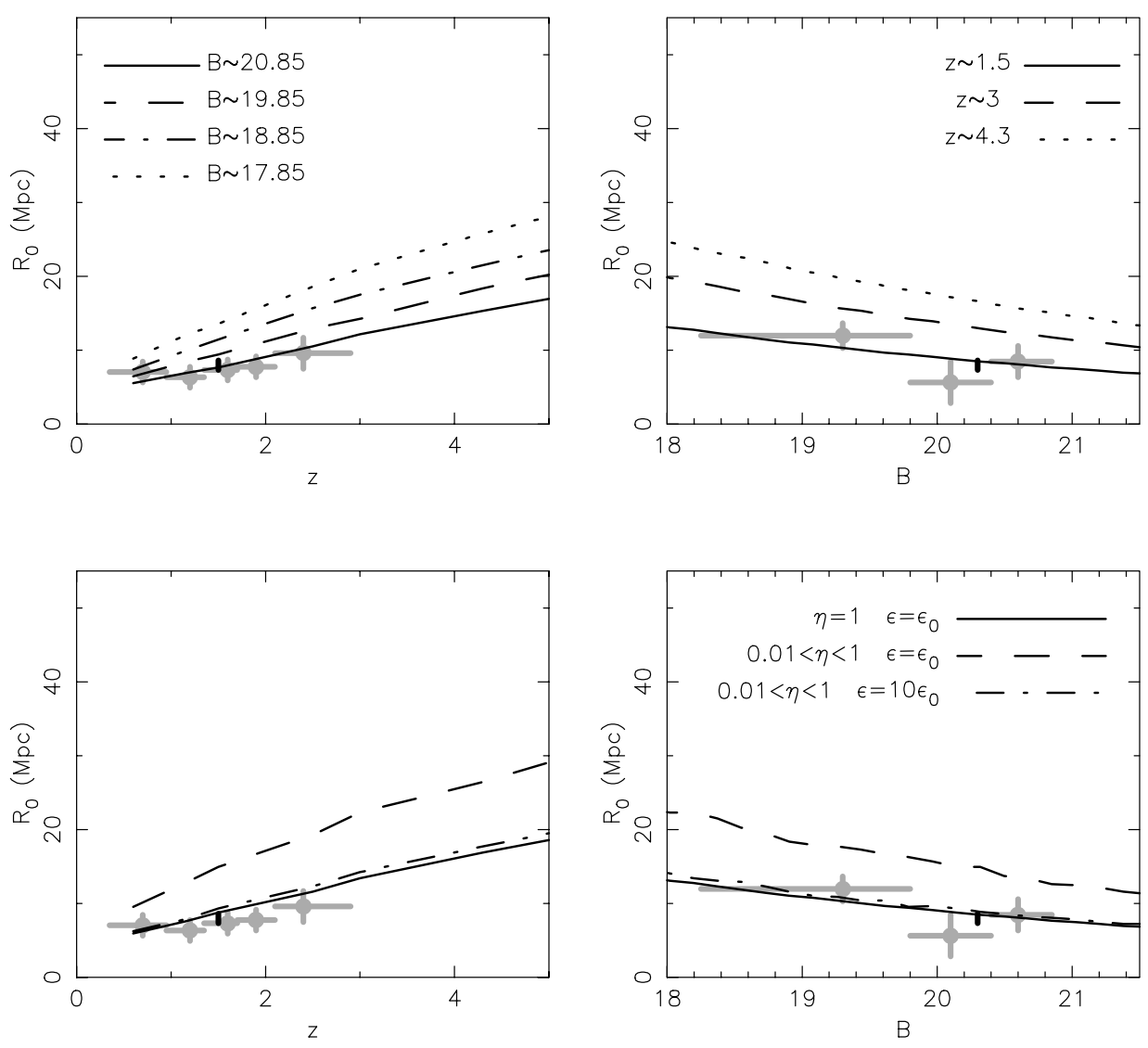

FIG. 2. - Correlation length $R_{0}$ [defined through $\xi_{q}\left(R_{0}\right)=1$ ] plotted as a function of redshift (left panels) and apparent magnitude (right panels). The data are from the $2 \mathrm{dF}$ quasar redshift survey (Croom et al. 2001a, 2002), with the gray error bars showing the correlation length from different subsamples, and the dark error bar at $z=1.5$ (left panels) and $B=20.3$ (right panels) showing the correlation length for the full sample. The theoretical model assumes $M_{\mathrm{bh}} \propto v_{c}^{5}$. In the top panels $\eta=1$ (case AI), and the curves are shown for different apparent magnitudes (left) and different redshifts (right). In the bottom panels we show curves corresponding to different choices for $\eta$ and $\epsilon$ (cases AII and AIII), assuming $B=20.3$ and $z=1.5$, respectively.

and at several apparent $B$-band magnitudes, ${ }^{3}$ as determined from the relation

$$
\begin{aligned}
B= & 5.5-2.5 \log _{10}\left(\frac{L_{B}}{L_{B, \odot}}\right)+5 \log _{10}\left(\frac{D_{L}}{10 \mathrm{pc}}\right) \\
& -2.5\left(1+\alpha_{\nu}\right) \log _{10}(1+z),
\end{aligned}
$$

where $\alpha_{\nu}=-0.5$ is the typical slope of the quasar power-law continuum (with a frequency dependence of the spectral flux $f_{\nu} \propto \nu^{\alpha_{\nu}}$ ), and $D_{L}$ is the luminosity distance. The magnitude limit of the $2 \mathrm{dF}$ quasar redshift survey is $B \sim 20.85$, and so the data should be compared with the model correlation function corresponding to $B \sim 20-21$ (solid and dashed lines in Fig. 1). Note that we are comparing correlation functions at a particular apparent magnitude with a correlation function computed from an observed sample having a range of apparent magnitudes brighter than $B \sim 20.85$. This approach is valid for two reasons. First, the steepness of the quasar luminosity function means that most of the quasars in the sample are near the magnitude limit, and second, there is little evolution in the correlation length with luminosity (see below). The bottom right panel of Figure 1 shows the $2 \mathrm{dF}$ correlation function generated from the entire quasar sample. These data are compared

\footnotetext{
${ }^{3}$ We refer to the Johnson $B$-magnitude, which for a typical quasar is related to the photographic $b$ band used in the $2 \mathrm{dF}$ quasar redshift survey by $B \approx b+0.06$ (Goldschmidt \& Miller 1998).
}

with a model correlation function computed at the median quasar redshift of the survey $(\langle z\rangle \sim 1.5)$. The $B=20.85$ prediction at this redshift is replotted in the other panels of Figure 1 to guide the eye (thick gray curves). We find that the correlation function depends more sensitively on redshift than on luminosity over the ranges under consideration.

\subsection{The Correlation Function for Models with $M_{\mathrm{bh}} \propto v_{c}^{5}$}

Variation of clustering with redshift and apparent magnitude is demonstrated more clearly in Figure 2. The top panels show the dependences of the clustering length $R_{0}$ [defined through the condition $\xi_{q}\left(R_{0}\right)=1$ ] for the fiducial case, where the SMBH mass scales with halo circular velocity and quasars shine at their Eddington limit (case AI). The correlation length is plotted for various values of apparent magnitude as a function of redshift (left panel) and various redshifts as a function of apparent magnitude (right panel). The data for the correlation length as a function of redshift were computed from a sample having $B \lesssim 20.85$ drawn from the $2 \mathrm{dF}$ quasar redshift survey (Croom et al. 2001a, 2002). The gray error bars show the values for $R_{0}$ in different redshift bins, while the correlation length measured for the whole sample (plotted at $z=1.5$ and $B=20.3$ in the left and right panels, respectively) is shown by the dark error bar. We find the predictions for $R_{0}$ to be consistent with observations. In particular, both the value of the clustering length and its slow evolution with redshift and apparent magnitude are consistent with current data. There is little 

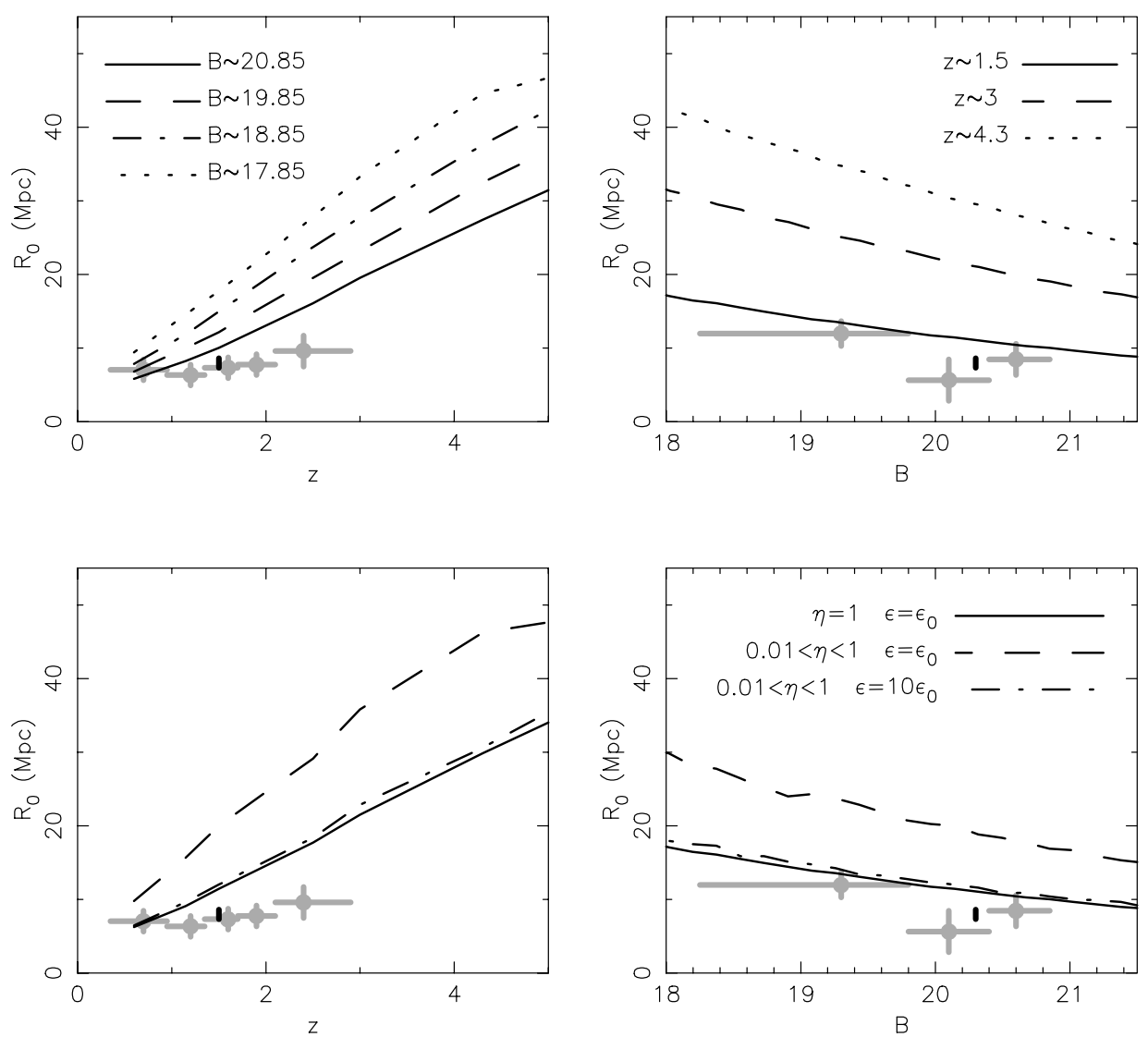

FIG. 3.-Correlation length $R_{0}$ as a function of redshift (left panels) and apparent $B$-band magnitude (right panels). The data are from the $2 \mathrm{dF}$ quasar redshift survey (Croom et al. 2001a, 2002), with the gray error bars showing the correlation length from different subsamples, and the dark error bars at $z=1.5$ (left panels) and $B=20.3$ (right panels) showing the correlation length for the full sample. The model assumes $M_{\mathrm{bh}} \propto M_{\text {halo. }}^{5 / 3}$. In the top panels $\eta=1$ (case BI), and the curves are shown for different apparent magnitudes (left panel) and different redshifts (right panel). In the bottom panels we show curves corresponding to different choices for $\eta$ and $\epsilon$ (cases BII and BIII), assuming $B=20.3$ and $z=1.5$, respectively.

variation of the clustering length with apparent magnitude over the range considered, although there is a tendency for brighter quasars to be more highly clustered, as was noted by Croom et al. (2002).

Note that the $K$-correction assumed in equation (9) is only valid out to $z \sim 3$, where Ly $\alpha$ absorption enters into the $B$ band. As a result, the extrapolations of redshift dependence to $z \gtrsim 3$ shown in Figures 2 and 3 do not include Ly $\alpha$ absorption in the calculation of the quasar luminosity. However, these extrapolations are included to illustrate the evolution of the clustering length at yet higher redshifts (as measured in bands redward of the redshifted Ly $\alpha$ line).

The bottom panels of Figure 2 show the corresponding dependence of the clustering length on redshift and apparent magnitude for quasars that shine at Eddington fractions $\eta$ lower than unity. Only the cases of $B=20.3$, corresponding approximately to the median quasar $B$-magnitude (left panel), and $z=1.5$ (right panel) are shown, and the curve corresponding to $\eta=1$ (case AI) is reproduced from the top panels for comparison (solid lines). Assuming the local normalization $\epsilon=\epsilon_{\mathrm{SIS}}$ and that $\eta$ ranges between 0.01 and 1 with a constant $d P_{\text {obs }} / d(\log \eta)$ (case AII), the typical quasar of apparent magnitude $B$ must be powered by a more massive $\mathrm{SMBH}$, and hence reside in a more massive galaxy than in the $\eta=1$ case. This results in a larger clustering length for quasars of a given apparent magnitude $B$. The resulting evolution, indicated by the dashed lines, appears to be incompatible with the data. The increase in $R_{0}$ can be compensated for by an increase in the normalization of the $M_{\mathrm{bh}}-M_{\text {halo }}$ relation. The dot-dashed curves show the result of increasing this normalization relative to local observations by a factor of $10\left(\epsilon=10 \epsilon_{\mathrm{SIS}}\right)$. This factor equals the inverse of the median of the distribution $d P / d(\log \eta)=$ const. The resulting curve (corresponding to case AIII) is very similar to our fiducial case $(\eta=1)$.

These results demonstrate that while a spread in the values of $\eta$ for different quasars results in the correlation function being measured over a wider range of halo masses, the correlation length is primarily sensitive to the characteristic halo mass, as specified by the characteristic value of $\eta$. Furthermore, the results demonstrate the important point that the correlation length measures the product of the normalization in the $M_{\mathrm{bh}}-M_{\text {halo }}$ relation and the median fraction of the Eddington luminosity at which quasars shine, $\eta_{\text {med }}$. With respect to the correlation function, this quantity $\epsilon \eta_{\text {med }}$ (which is the same in cases AI and AIII) is more fundamental to the correlation function than the quasar lifetime, since it predicts the correlation length independent from consideration of quasar number counts. It is important to note that since the normalization of the local $M_{\mathrm{bh}^{-}}$ $M_{\text {halo }}$ relation is estimated ( $\epsilon=\epsilon_{\text {SIS }}$; Ferrarese 2002), the value of the quasar correlation length at low redshift implies that quasars spend most of their bright phase shining near their Eddington luminosity.

\subsection{The Correlation Function for Models with $M_{\mathrm{bh}} \propto M_{\text {halo }}^{5 / 3}$}

We next examine whether the correlation function observed by the $2 \mathrm{dF}$ survey can be used to constrain the evolution of the $M_{\mathrm{bh}}-M_{\text {halo }}$ relation. In the previous subsection, we considered 
the case of a SMBH mass that scales with the fifth power of the circular velocity independent of redshift (eq. [7], case A). The alternative case $\mathrm{B}$ example postulates that the $\mathrm{SMBH}$ mass has a redshift-independent scaling with the halo mass rather than the halo circular velocity (eq. [8]). The top panels of Figure 3 plot the correlation length in this case with $\eta=1$ and $\epsilon=\epsilon_{\mathrm{SIS}}$ (case BI). Both the evolution of the correlation length with redshift for different apparent magnitudes (left panel) and the variation of correlation length with apparent magnitude at various redshifts (right panel) are shown. The case B $M_{\mathrm{bh}}-M_{\text {halo }}$ relation results in a correlation length that varies significantly more with redshift than the data require. The bottom panels show examples with values of $\eta$ that differ from unity. Only the cases of $B=20.3$ (left panel) and $z=1.5$ (right panel) are shown, and the result assuming $\eta=1$ (case BI) is reproduced from the top panels for comparison (solid lines). If $\epsilon=\epsilon_{\mathrm{SIS}}$, and $\eta$ ranges between 0.01 and 1 with $d P_{\text {obs }} / d \eta \propto$ const (case BII), then the typical quasar of apparent magnitude $B$ must have a larger clustering length (dashed lines). This can be compensated for by increasing the normalization of the $M_{\mathrm{bh}^{-}}$ $M_{\text {halo }}$ relation by a factor of $10\left(\epsilon=10 \epsilon_{\mathrm{SIS}}\right.$, case BIII), as considered before (dot-dashed lines).

The rapid evolution with redshift that results from a case B $M_{\mathrm{bh}}-M_{\text {halo }}$ relation could be diminished if the luminosities of quasars were a higher fraction of their Eddington limit at higher redshift. However, we have seen that the locally observed normalization of the $M_{\mathrm{bh}}-M_{\text {halo }}$ relation requires most presentday quasars to be shining near their Eddington luminosity. Quasars at high redshift would therefore have to be superEddington, shining at $\sim \eta \sim(1+z)^{5 / 2}(\eta \sim 30$ at $z \sim 3)$ in order to reproduce the observed slow evolution of the clustering length. This requirement on $\eta$ may be somewhat relaxed in models having $\epsilon>\epsilon_{\mathrm{SIS}}$, as is discussed in the next section.

\section{CONSTRAINING $\eta$ AND $\epsilon$ USING THE QUASAR CORRELATION FUNCTION}

The results in the preceding sections have been presented for fiducial models with $(\epsilon, \eta)=\left(\epsilon_{\mathrm{SIS}}, 1\right),\left(\epsilon_{\mathrm{SIS}}, 0.1\right)$, and $\left(10 \epsilon_{\mathrm{SIS}}, 0.1\right)$. It was demonstrated that the correlation function is primarily dependent on the product between $\epsilon$ and the median value of $\eta$, namely $\epsilon \eta_{\text {med }}$. This raises the possibility that $\epsilon \eta_{\text {med }}$ could be constrained using correlation function data. We have computed the evolution of the correlation length $R_{0}(z)$ for a set of models having a range of values for the product $\epsilon \eta_{\text {med }} \equiv f_{\epsilon} \epsilon_{\mathrm{SIS}} \eta_{\text {med }}$ and calculated the reduced $\chi^{2}$ statistics as well as the confidence with which a model is excluded by the $2 \mathrm{dF}$ data, $P\left(\chi^{2}\right)$. In Figure 4 we plot the resulting $P\left(\chi^{2}\right)$ as a function of $f_{\epsilon} \eta_{\text {med }}$ (dark lines).

The results suggest that case A models (solid lines) favor $f_{\epsilon} \eta_{\text {med }} \sim 1-5$, indicating that $\epsilon$ is a factor of a few larger than $\epsilon_{\mathrm{SIS}}$ for $\eta \sim 1$, as would be expected from the universal NFW96 profile, or that $\eta_{\text {med }}$ has a maximum value of a few tenths. If $\epsilon=\epsilon_{\mathrm{S} 02}$, case A requires $\eta_{\text {med }} \sim 0.08$. Figure 4 shows that case $\mathrm{B}$ models (dashed lines) are more restricted, requiring $f_{\epsilon} \eta_{\text {med }} \sim$ 10-30. This implies that $\epsilon=\epsilon_{\mathrm{SIS}}$ and $\epsilon=\epsilon_{\mathrm{NFW}}$ cannot be accommodated, since the required values of $\eta_{\text {med }} \gtrsim 10$ and $\eta_{\text {med }} \gtrsim 3$, respectively, are considered unphysical in most accretion models (Nityananda \& Narayan 1982; see, however, Ruszkowski \& Begelman 2003). The more extreme case of $\epsilon=\epsilon_{\mathrm{S} 02}$ may be marginally accommodated by case B; however, even in this case the quasars must shine somewhat in excess of their Eddington limit.

Figure 4 also shows $P\left(\chi^{2}\right)$ as a function of $f_{\epsilon} \eta_{\text {med }}$ for a hypothetical future survey that would yield the same evolution of $R_{0}$ with redshift, but with errors that are smaller by a factor of

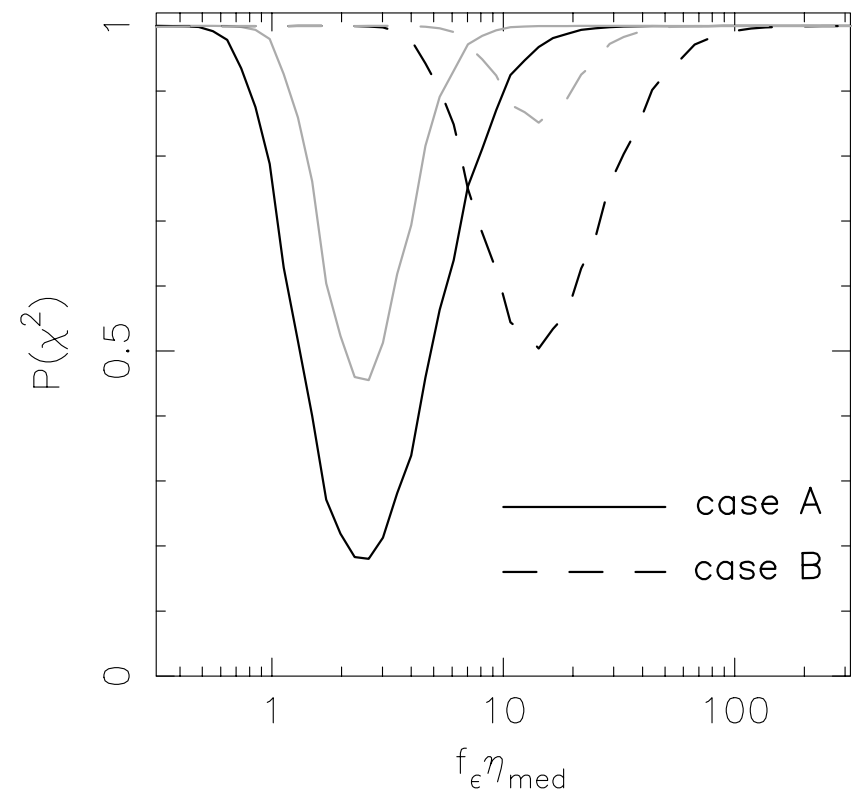

FIG. 4.-Probability $P\left(\chi^{2}\right)$ of case A and case B models as a function of $f_{\epsilon} \eta_{\text {med }}$. The dark lines correspond to fits to the redshift-dependent correlation length $R_{0}(z)$ measured by the $2 \mathrm{dF}$ quasar redshift survey (Croom et al. 2001a). The light lines show results for a hypothetical larger future sample whose errors on $R_{0}$ are reduced by a factor of $\sqrt{2}$ relative the $2 \mathrm{dF}$ quasar survey.

$\sqrt{2}$ (light lines). Future surveys will better constrain the gradient $d R_{0} / d z$ and help discriminate further between different models of the $M_{\mathrm{bh}}-M_{\text {halo }}$ relation.

Our discussion so far has been independent of the quasar luminosity function. In the next section we discuss how the luminosity function can be used to determine the redshift evolution of the quasar lifetime.

\section{THE LUMINOSITY FUNCTION AND QUASAR LIFETIME}

In the previous sections we have demonstrated that the evolution of the $M_{\mathrm{bh}}-M_{\text {halo }}$ relation with redshift can be constrained directly from the quasar correlation function. We have shown that the critical parameter determining the SMBH mass is the halo circular velocity rather than the halo mass. We reiterate that this result does not rely on the luminosity function of quasars. On the other hand, the number counts of quasars are proportional to the quasar lifetime. Therefore, the quasar lifetime and its evolution with redshift can be determined by comparing the observed quasar number counts with the SMBH mass function. The latter can be obtained from the Press-Schechter mass function (Press \& Schechter 1974) of dark matter halos (with the improvement of Sheth \& Tormen 1999) combined with the $M_{\mathrm{bh}}-M_{\text {halo }}$ relation.

In this section we show that two assumptions can be added to the simple model presented in the previous sections that enable the model to also reproduce the quasar luminosity function. These assumptions are that quasar activity is associated with major mergers of galaxies (e.g., Kauffmann \& Haehnelt 2000; Wyithe \& Loeb 2003) and that the quasar lifetime is set by the dynamical time of a galactic disc.

A simple model that attributes quasar activity to major mergers, assumes an $M_{\mathrm{bh}}-M_{\text {halo }}$ relation that is described by equation (7), and sets the quasar lifetime equal to the dynamical time of a galactic disk accurately reproduces the entire optical and X-ray luminosity functions of quasars at redshifts 

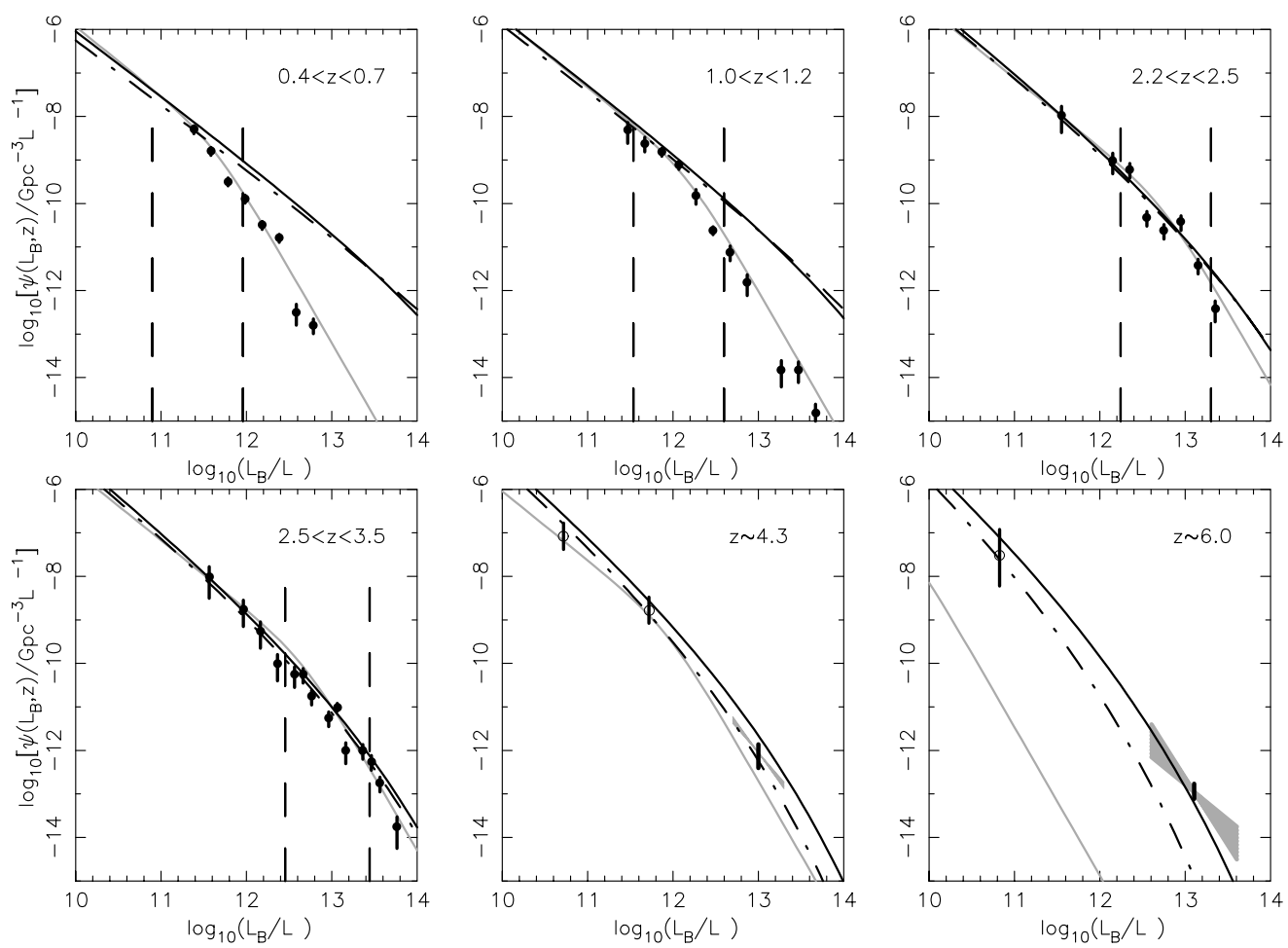

FIG. 5.-Comparison of the model and observed quasar luminosity functions at various redshifts. The data points at $z \lesssim 4$ are summarized in Pei (1995), while the light lines show the double power-law fit to the 2dF quasar luminosity function (Boyle et al. 2000). At $z \sim 4.3$ and $z \sim 6.0$ the data are from Fan et al. (2001a, $2001 \mathrm{~b}, 2003)$. The gray regions show the $1 \sigma$ range of logarithmic slope $([-2.25,-2.75]$ at $z \sim 4.3$ and $[-1.6,-3.1]$ at $z \sim 6)$, and the vertical bars show the uncertainty in the normalization. The open circles show data points converted from the X-ray luminosity function (Barger et al. 2003) of low-luminosity quasars using the median quasar spectral energy distribution (Elvis et al. 1994). The model luminosity functions shown correspond to case AI (solid curves) and a model assuming case B evolution with $\epsilon=\epsilon_{\mathrm{S} 02}, \eta=1$, and a constant lifetime of $t_{q}=10^{7} \mathrm{yr}$ (dot-dashed curves). The dashed pairs of vertical lines show the range corresponding to apparent $B$-magnitudes between 18.25 and 20.85 .

between 2 and 6 (Wyithe \& Loeb 2003). At lower redshifts $(z \lesssim 2)$, this prescription correctly predicts the luminosity function at luminosities below the characteristic break (Boyle et al. 2000). We reproduce this model here, but in contrast to Wyithe \& Loeb (2003) we have assumed the quasar activity to take place in the larger SMBH before coalescence of the SMBH binary formed during the merger (Volonteri et al. 2003). We also compute the corresponding model luminosity function assuming the case B $M_{\mathrm{bh}}-M_{\text {halo }}$ relation, in addition to case A.

Figure 5 shows the comparison of this model with observations. Specifically, we show the fiducial case AI (which corresponds to the model presented in Wyithe \& Loeb 2003) using equation (7) with $\epsilon=\epsilon_{\text {SIS }}$ and $\eta=1$ (solid curves). We have shown that case AI describes the observed quasar correlation function (see Figs. 1 and 2). However, as discussed in $\S 5$, case B models for the $M_{\mathrm{bh}}-M_{\text {halo }}$ relation also provide acceptable fits to $R_{0}(z)$ as long as $\epsilon \gtrsim 10 \epsilon_{\text {SIS }}$ and $\eta \sim 1$. Therefore, a corresponding luminosity function model was also generated for this case assuming a constant lifetime of $t_{q}=10^{7} \mathrm{yr}$. We have plotted this model in Figure 5 (dot-dashed lines).

We find that our fiducial model (case AI; Fig. 5, solid lines) with a quasar lifetime that equals the dynamical time of a galactic disk reproduces the observed luminosity function over a wide range of redshifts and luminosities (see Wyithe \& Loeb 2003). Our second model (case $\mathrm{B}, \epsilon \gtrsim 10 \epsilon_{\mathrm{SIS}}, \eta=1, t_{q}=10^{7}$; Fig. 5, dot-dashed lines) also reproduces the observed luminosity function at $z \lesssim$ 4.5. However, at $z \sim 6$ this model underestimates the observed number counts by 2 orders of magnitude. Case B evolution for the $M_{\mathrm{bh}}-M_{\text {halo }}$ relation is therefore challenged by the existence of the highest redshift quasars, which would imply a quasar lifetime that exceeds the age of the universe at $z \sim 6$. Moreover, given the Salpeter (1964) time of $\sim 4 \times 10^{7} \mathrm{yr}$, the SMBHs powering these quasars would grow to 25 times their initial mass during their lifetime. Provided that quasar activity is triggered by major mergers, the requirement that the $M_{\mathrm{bh}}-M_{\text {halo }}$ relation reproduce the observed evolution in quasar clustering together with the observed evolution in the number counts of quasars therefore implies case A evolution and a quasar lifetime that is set by the dynamical time of the host galaxy. We reinforce the important qualitative result that since the evolution of quasar correlations requires SMBHs to take a larger fraction of the halo mass at high redshift (case A; see eq. [7]), the observed evolution in the number counts requires a quasar lifetime that is shorter at higher redshifts, scaling approximately as the dynamical time of a dark matter halo $\left[\propto(1+z)^{-3 / 2}\right]$.

We point out that the model luminosity functions fail to reproduce the rapid drop in the density of luminous quasars seen at low redshift. This drop is thought to be due to the consumption of cold gas in galaxies by star formation (e.g., Kauffmann \& Haehnelt 2000) and the inhibition of accretion onto massive halos at late times (e.g., Scannapieco \& Oh 2004). However, most of the quasars in the $2 \mathrm{dF}$ quasar redshift survey have $B$-magnitudes that lie below the characteristic break in the luminosity function at low redshift (Boyle et al. 2000; Croom et al. 2001a). This can be seen in Figure 5, where the vertical dashed lines show the luminosities bracketing the apparent magnitude range $18.25<B<20.85$. Since the model correctly predicts the luminosity function within most of this luminosity range at all redshifts, we are justified in deriving combined constraints from the luminosity and correlation functions of quasars. 


\section{DO BRIGHT LOW-REDSHIFT QUASARS SHINE NEAR THEIR EDDINGTON LUMINOSITY?}

In $\oint 4$ we demonstrated that the $2 \mathrm{dF}$ quasar sample has a correlation length that is consistent with the local $M_{\mathrm{bh}}-M_{\text {halo }}$ relation and that quasars shine near their Eddington luminosity. In addition, it was shown that this consistency extends to both bright and faint subsamples of quasars. The brightest $2 \mathrm{dF}$ quasars $(18.1 \leq B \leq 19.9)$ presented by Croom et al. (2002) are mostly brighter than the characteristic break luminosity in the differential quasar number counts (e.g., Boyle et al. 2000). These bright quasars have a lower abundance than expected from merging halos at $z \lesssim 2$ (see Fig. 5). Either the lifetime (or occupation fraction) of bright quasars is lower than expected at $z \lesssim 2$, or else these quasars shine well below their Eddington limit. Figure 2 indicates that the slow dependence of $R_{0}$ with $B$ reported by Croom et al. (2002) is consistent with the expected trend if all quasars shine near their Eddington luminosity. This consistency suggests that bright quasars are underrepresented at $z \lesssim 2$ because of a reduced quasar lifetime (or a reduced occupation fraction) rather than a reduced value of $\eta$ during their brightest phase.

\section{DISCUSSION}

In this paper we have demonstrated that the relationship between the mass of a SMBH and its host dark matter halo is the fundamental quantity that determines the clustering of quasars. In particular, the $M_{\mathrm{bh}}-M_{\text {halo }}$ relation and its evolution with redshift can be determined directly from the evolution in the correlation length of quasars, independent of consideration of the quasar luminosity function or assumptions about the quasar lifetime. Beginning with the locally observed $M_{\mathrm{bh}}-M_{\text {halo }}$ relation, we have shown that the observed correlation length of quasars in the $2 \mathrm{dF}$ quasar redshift survey is consistent with SMBHs that shine at $\sim 10 \%-100 \%$ of their Eddington luminosity during their bright quasar episode. Moreover, the evolution of the clustering length with redshift is consistent with a SMBH mass whose redshift-independent scaling is with the circular velocity of the host dark matter halo (see eq. [7]). In contrast, it appears that a relation for the SMBH mass whose redshift-independent scaling is with the mass of the host dark matter halo (eq. [8]) would have resulted in too much evolution of the clustering length with redshift.

The portion of the $2 \mathrm{dF}$ quasar survey from which the data used in this study were drawn contains $\sim 10^{4}$ quasars. Upon completion, the SDSS will have spectra for 10 times this number of quasars, spread over a larger redshift range (York et al. 2000). This will allow more accurate determination of the correlation length and its evolution and hence provide more accurate constraints on the SMBH population. The selection of quasars for the spectroscopic survey of the SDSS at $z<3$ is restricted to $i^{*}<19$. Thus, most of the low-redshift SDSS quasars will fall at luminosities that are brighter than the characteristic break in the luminosity function (e.g., Boyle et al. 2000). These bright quasars have a lower abundance than expected from the number of halos merging at $z \lesssim 2$. The reason for this low abundance may be either that the lifetime (or occupation fraction) of bright quasars is lower than expected at $z \lesssim 2$, or, alternatively, that the fraction of the Eddington limit at which the quasars shine is small. The $2 \mathrm{dF}$ quasar sample straddles the luminosity function break. We have shown that the similarity in the clustering statistics of subsamples of quasars having different ranges in luminosity suggests that the lifetime (or occupation fraction) of bright quasars is lower than expected at $z \lesssim 2$, but that these quasars also shine near their Eddington luminosity. Measurements of the correlation length as a function of quasar luminosity in SDSS can help further distinguish between these two possibilities.

As we have shown in a previous paper (Wyithe \& Loeb 2003), feedback-regulated growth of SMBHs during active quasar phases implies $M_{\mathrm{bh}} \propto v_{c}^{5}$, consistent with the inferred relation for galactic halos in the local universe (Ferrarese 2002). Assuming $\eta=1$, as suggested by observations of both low- and highredshift quasars (Floyd 2004; Willott et al. 2003; Shankar et al. 2004), we have shown that only $\sim 7 \%$ of the Eddington luminosity output needs to be deposited into the surrounding gas in order to unbind it from the host galaxy over the dynamical time of the surrounding galactic disk. The power-law index of 5 in the $M_{\mathrm{bh}}-v_{c}$ relation is larger than the values of 4-4.5 inferred from the local relation between $M_{\mathrm{bh}}$ and the stellar velocity dispersion $\sigma_{*}$ (Merritt \& Ferrarese 2001; Tremaine et al. 2002), the difference originating from the observation that the $v_{c}-\sigma_{*}$ relation is shallower than linear for stellar bulges embedded in cold dark matter halos (Ferrarese 2002). Feedback-regulated growth also implies that the $M_{\mathrm{bh}}-v_{c}$ relation should be independent of redshift, as implied by the slow evolution in the clustering length of quasars.

A very simple model can therefore be constructed to describe the correlation length of quasars and its evolution in the $2 \mathrm{dF}$ survey, as well as the number counts of quasars out to very high redshift $(z \sim 6)$. The model includes four simple assumptions, but no free parameters: (1) the locally observed $M_{\mathrm{bh}^{-}}$ $M_{\text {halo }}$ relation extends to high redshifts through equation (7) with the SMBH mass scaling as halo circular velocity to the 5th power; (2) SMBHs shine near their Eddington luminosity with a universal spectrum (Elvis et al. 1994) during luminous quasar episodes; (3) quasar episodes are associated with major galaxy mergers; and (4) the quasar lifetime is set by the dynamical time of the host galaxy.

The assumption of a SMBH mass that scales with halo circular velocity independently of redshift is supported by the observations of Shields et al. (2003) that there is no evolution in the $M_{\mathrm{bh}}-\sigma_{*}$ relation out to $z \sim 3$. The assumption that quasars shine near their Eddington limit is supported by observations of high- and low-redshift quasars (Floyd 2004; Willott et al. 2003; Shankar et al. 2004). Overall, the sample of available data on the clustering properties and the number counts of quasars is most readily explained by a quasar lifetime that is set by the dynamical time of the host galaxy rather than by the Salpeter (1964) $e$-folding time for the growth of its mass.

This work was supported in part by NASA grant NAG 513292 and by NSF grants AST-0071019 and AST-0204514 (for A. L.). J. S. B. W. wishes to thank Princeton University Observatory for their hospitality during the course of this work.

\section{REFERENCES}

Barger, A. J., Cowie, L. L., Capak, P., Alexander, D. M., Bauer, F. E., Brandt, W. N., Garmire, G. P., \& Hornschemeier, A. E. 2003, ApJ, 584, L61

Barkana, R., \& Loeb, A. 2001, Phys. Rep., 349, 125

Boyle, B. J., Shanks, T., Croom, S. M., Smith, R. J., Miller, L., Loaring, N., \& Heymans, C. 2000, MNRAS, 317, 1014

Croom, S. M., Boyle, B. J., Loaring, N. S., Miller, L., Outram, P. J., Shanks, T., \& Smith, R. J. 2002, MNRAS, 335, 459

Croom, S. M., Shanks, T., Boyle, B. J., Smith, R. J., Miller, L., Loaring, N. S., \& Hoyle, F. 2001a, MNRAS, 325, 483

Croom, S. M., Smith, R. J., Boyle, B. J., Shanks, T., Loaring, N. S., Miller, L., \& Lewis, I. J. 2001b, MNRAS, 322, L29 
Elvis, M., et al. 1994, ApJS, 95, 1

Fan, X., et al. 2001a, AJ, 121, 54

2001b, AJ, 122, 2833

2003, AJ, 125,1649

Ferrarese, L. 2002, ApJ, 578, 90

Floyd, D. J. E. 2004, in Carnegie Observatories Astrophysics Series, Vol. 1: Coevolution of Black Holes and Galaxies, ed. L. C. Ho (Cambridge: Cambridge Univ. Press), 16

Goldschmidt, P., \& Miller, L. 1998, MNRAS, 293, 107

Haiman, Z., \& Hui, L. 2001, ApJ, 547, 27

Jakobsen, P., Jansen, R., A., Wagner, S., \& Reimers, D. 2003, A\&A, 397, 891

Kauffmann, G., \& Haehnelt, M. 2000, MNRAS, 311, 576 2002, MNRAS, 332, 529

Magorrian, J., et al. 1998, AJ, 115, 2285

Martini, P. 2004, in Carnegie Observatories Astrophysics Series, Vol. 1: Coevolution of Black Holes and Galaxies, ed. L. C. Ho (Cambridge: Cambridge Univ. Press), 170

Martini, P., \& Weinberg, D. H. 2001, ApJ, 547, 12

McLure, R. J., \& Dunlop, J. S. 2002, MNRAS, 331, 795

Merritt, D., \& Ferrarese, L. 2001, ApJ, 547, 140

Mo, H. J., \& White, S. D. M. 1996, MNRAS, 282, 347

Navarro, J. F., Frenk, C. S., \& White, S. D. M. 1996, ApJ, 462, 563 (NFW96)
Nityananda, R., \& Narayan, R. 1982, MNRAS, 201, 697

Pei, Y. C. 1995, ApJ, 438, 623

Press, W. H., \& Schechter, P. 1974, ApJ, 187, 425

Ruszkowski, M., \& Begelman, M. C. 2003, ApJ, 586, 384

Salpeter, E. E. 1964, ApJ, 140, 796

Scannapieco, E., \& Barkana, R. 2002, ApJ, 571, 585

Scannapieco, E., \& Oh, S. P. 2004, ApJ, 608, 62

Seljak, U. 2002, MNRAS, 334, 797

Shankar, F., Salucci, P., Granato, G. L., De Zotti, G., \& Danese, L. 2004, MNRAS, in press (astro-ph/0405585)

Sheth, R. K., Mo, H. J., \& Tormen, G. 2001, MNRAS, 323, 1

Sheth, R. K., \& Tormen, G. 1999, MNRAS, 308, 119

Shields, G. A., Gebhardt, K., Salviander, S., Wills, B. J., Xie, B., Brotherton,

M. S., Yuan, J., \& Dietrich, M. 2003, ApJ, 583, 124

Silk, J., \& Rees, M. J. 1998, A\&A, 331, L1

Spergel, D. N., et al. 2003, ApJS, 148, 175

Tremaine, S., et al. 2002, ApJ, 574, 740

Volonteri, M., Haardt, F., \& Madau, P. 2003, ApJ, 582, 559

Willott, C. J., McLure, R. J., \& Jarvis, M. J. 2003, ApJ, 587, L15

Wyithe, J. S. B., \& Loeb, A. 2003, ApJ, 595, 614

York, D. G., et al. 2000, AJ, 120, 1579

Yu, Q., \& Tremaine, S. 2002, MNRAS, 335, 965 